\title{
MUJERES, APRENDIZAJE DIALÓGICO Y TRANSFORMACIÓN SOCIAL
}

\author{
Carmen Elboj Saso \\ Ainhoa Flecha Fernández
}

RESUMEN. En el contexto de las sociedades informacionales, la mujer sin formación académica sufre una doble exclusión: la primera a causa de su condición de mujer y, la segunda, por su falta de conocimientos académicos en un contexto en el que se prioriza cada vez más el nivel de instrucción en el ámbito de la educación formal.

Este artículo trata y profundiza en las posibilidades de transformación que encierran la voz de aquellas mujeres que tradicionalmente han estado excluidas de la educación y, por lo tanto, del aprendizaje a lo largo de la vida y de la participación social.

El tema de la mujer ha sido tratado desde el mundo académico dentro del análisis de las desigualdades sociales y educación. Por un lado, uno de los temas poco analizados es el papel de la educación no formal en la vida de las mujeres no universitarias. La formación acostumbra a basarse en concepciones académicas de la educación, a pesar de que actualmente en la sociedad de la información está demostrado que la educación depende cada vez más de la correlación entre lo que se aprende en las aulas y lo que se aprende fuera de los ámbitos académicos. Por otro lado, aunque el tema de la mujer se está trabajando, no se tiene en consideración la situación de las mujeres no universitarias.

En este sentido, creemos que con el tratamiento de este tema no sólo coincidimos con las líneas de investigación de la comunidad científica internacional sino que, a la vez, contribuimos a la formulación de nuevas propuestas de participación y transformación social a través del aprendizaje dialógico y del feminismo dialógico en el que se basa una Educación de Personas Adultas de orientación dialógica.

ABSTRACT. In the context of information societies, non academic women find themselves in a situation of double exclusion: firstly due to their condition as women and secondly due to their lack of academic knowledge in a context in which the level of formal education is more and more important.

This article deals with and studies in depth the transformation possibilities that enclose the voice of those women who have traditionally been excluded from education, and therefore from long life learning and social participation.

The theme of women has been treated from the academic world within the analysis of social inequalities and education. On the one hand, one of the issues which have been poorly studied is the role of non formal education in the life of non academic women. Education is usually based on academic conceptions of education, whilst currently in information society it is being proved that education depends more and more on the correlation between what we learn in the classrooms and what is learned 
out of the academic contexts. On the other hand, although the issue on women is being dealt with, the situation of non academic women in not being taken into consideration.

In this sense, we believe that with the treatment of this subject not only do we agree with the lines of research from international scientific community, but also do we contribute to the formulation of new proposals of social participation and education through dialogic learning and dialogic feminism in which dialogic adult education is based.

\section{Educación de mujeres no académicas en la Sociedad de la Información}

Los cambios sociales y culturales que se están produciendo en el paso a la sociedad de la información vienen definidos por lo que denominamos el giro dialógico (Flecha, Gómez, Puigvert 2001). Por una parte, nos encontramos ante sociedades que se vuelven dialógicas puesto que las tradiciones, maneras de hacer y demás componentes del horizonte de nuestro mundo de la vida se ven continuamente cuestionados y nos confrontan a la necesidad de consensuar y dialogar. La legitimización de las diferentes instituciones sociales, entre ellas los centros de Educación de Personas Adultas, pasan por este consenso. Por otra parte, la teoría y metodología de las ciencias sociales también participa de este giro dialógico, ya que debe plantearse el análisis riguroso de los cambios que estamos viviendo y hacerlo desde la consideración de la acción de los diferentes agentes sociales y su capacidad de interpretar y dar sentido a sus propias acciones.

En el contexto de las sociedades informacionales la mujer sin estudios superiores sufre una doble exclusión: la primera, a causa de su condición de mujer y, la segun$\mathrm{da}$, por su falta de credenciales y conocimientos académicos en un contexto en el que se prioriza cada vez más el nivel de instrucción. La Educación de Personas Adultas se enfrenta al reto de dar respuesta a las necesidades de las mujeres sin titulación para que puedan hacer frente a los requerimientos que les exige la sociedad actual.

Tradicionalmente las mujeres de los sectores populares se han visto excluidas del sistema educativo. A las barreras familiares, económicas y culturales que ha construido la sociedad hemos de sumar las de un sistema educativo que ha obviado la inteligencia y las habilidades que las mujeres han desarrollado en la práctica en su vida cotidiana y en el curso de su socialización, tratándolas de incompetentes y entorpeciendo así su aprendizaje y su transformación personal.

Una prueba de ello, son los resultados que presentan algunas de las investigaciones que se han realizado recientemente en torno al nivel de participación de la población adulta en procesos de formación, que muestran cómo las mujeres populares continúan excluidas de un gran número de ofertas culturales y educativas'. Se trata del colectivo que más participa en los programas de formación básica, pero no podemos decir lo mismo con relación a los programas de formación ocupacional y continua. Ello se debe, por una parte, a los nuevos requerimientos del mercado laboral, que

1. CREA. 1996. Participació i no participació en la formació de personas adultes a Catalunya. Unesco Insitute of Education y Departament de Benestar Social de la Generalitat de Catalunya. 
exige a los trabajadores y las trabajadoras tener una cualificación cada vez mayor $y$, por otra parte, a los prejuicios que existen en torno a las capacidades y posibilidades de las mujeres sin cualificación para insertarse en la economía formal y en los procesos de participación social y cultural en general.

En este contexto se presenta el aprendizaje dialógico, uno de los pilares del feminismo dialógico que se configura en la actual sociedad de la información como una clave fundamental para sustentar una educación superadora de la exclusión que sufren las mujeres no académicas.

La función de la educación en la sociedad de la información cambia sustancialmente de lo que era en la sociedad industrial. Desde nuestra postura defendemos la función transformadora de la educación y para ello debemos empezar reconociendo los aprendizajes adquiridos a lo largo de la vida y la voz de estas mujeres. Tener en cuenta estas premisas supone (Elboj, Flecha 2000):

1. Acreditación de la experiencia previa mediante políticas APEL. Es decir, desarrollar políticas de acreditación y evaluación de los aprendizajes adquiridos a través de la experiencia. De esta forma, se facilita el acceso a la educación y a la formación de muchas personas que carecen de titulaciones académicas como es el caso de las mujeres sin titulación superior.

2. Partir de la experiencia en todos los aprendizajes. Desde nuestra postura defendemos el currículum específico de personas adultas. Si partimos de la premisa de que los nuevos conocimientos y habilidades deben estar basados en la experiencia previa será imprescindible la participación de las mujeres en el diseño y confección de la oferta formativa.

\section{Feminismo dialógico: Inclusión de todas las mujeres}

Las mujeres hemos promovido y conseguido transformaciones igualitarias en las relaciones de género que desmienten las concepciones sociológicas funcionalistas y estructuralistas que negaban sus posibilidades y las concepciones antisociológicas postmodernas que negaban no sólo sus posibilidades sino también su conveniencia. El siglo XX ha sido calificado por algunas autoras y autores (Castells 1997-1998/19961997, Beck 1998a/1986, 1998b/1997; Beck y Beck-Gernsheim 1998/1990, Giddens , 1995a/1992, 1995b/1991; Touraine 1997; Subirats 1998) como el de la revolución de las mujeres. Gran parte de esas transformaciones se han iniciado y han encontrado rápido eco en las escuelas desmintiendo así también el intelectualmente mediocre y políticamente conservador modelo de la reproducción.

En la actualidad el movimiento feminista sigue jugando un papel fundamental para conseguir una sociedad más igualitaria y más democrática, aunque debe dar un paso más y superar el elitismo académico que le ha caracterizado hasta la actualidad. El feminismo dialógico es la apuesta por un feminismo universal y la lucha por la igualdad de oportunidades de todas las mujeres respetando las diferentes identidades y experiencias.

La propuesta del feminismo dialógico es fruto del trabajo conjunto entre mujeres académicas y no académicas. Este artículo no podría haber sido posible sin las apor- 
taciones de las mujeres sin estudios superiores de FACEPA². Lídia Puigvert (2001), junto a las Otras mujeres ${ }^{3}$, hace aportaciones sobre la ampliación del feminismo, reivindicando la salida de la situación de invisibilidad y exclusión en la que se encuentran mujeres que carecen del nivel de estudios exigido por la cultura dominante, que implica no poder realizar aportaciones a la teoría feminista. Las reivindicaciones de las mujeres sin título universitario son precisamente las que nos muestran que el feminismo no pertenece a una elite académica sino que es responsabilidad de todas las mujeres.

El camino a recorrer es largo, puesto que la mayoría de las mujeres es excluida del discurso público por parte de la minoría que tiene título universitario, que no sólo lo hegemoniza sino que también lo monopoliza. Esa minoría exige una determinada cuota o la paridad entre hombres y mujeres en los cargos públicos o en la mesa de un congreso pero no se da cuenta de que la participación de las mujeres no universitarias en esos mismos marcos es usualmente igual al $0 \%$. Cuando reivindican la igualdad salarial habitualmente piensan en referencia a sus colegas hombres, pero no en las mujeres que ellas mismas tienen en sus casas haciendo la limpieza.

La exclusión de las Otras mujeres se ha producido por la priorización dada a los saberes académicos propios de la cultura dominante. La gran mayoría de feministas y mujeres académicas construyen barreras a la participación a aquellas Otras mujeres que no poseen un título universitario. Con ello, se pasan por alto las capacidades que éstas han desarrollado en contextos no-académicos, y las luchas que están llevando a cabo en sus vidas diarias. Muchas Otras mujeres no se reconocen como feministas, si bien son más feministas y coherentes en sus prácticas diarias que muchas otras que sí se declaran feministas y que, una vez han conseguido acceder a una educación superior y a un buen puesto de trabajo regulado, se olvidan de reivindicar los mismos derechos para las mujeres de la limpieza que están trabajando para ellas. Cuando hablan de feminismos diferentes se alude a las mujeres afroamericanas, magrebíes o gitanas, pero de nuevo el discurso público de éstas es hegemonizado o monopolizado por su minoría universitaria.

A los centros de Educación de Personas Adultas acuden muchas de esas mujeres de la limpieza y, en general, mujeres no universitarias. Cuando ese centro tiene una orientación dialógica, las Otras mujeres se constituyen en sujetos de su propia transformación haciendo realidad los análisis y propuestas de la sociología general y la sociología de la educación de orientación comunicativa (Flecha 1997; Freire 1997/1995; Giddens 1993/1991; Habermas 1987-1989/1981; Subirats 1998).

Es importante que las voces de las Otras mujeres lleguen a espacios de debate en los que se crea la teoría feminista. Hemos tenido que esperar hasta el año 2001 para ver mujeres académicas y no académicas dialogando de forma igualitaria sobre el feminismo del futuro mediante la inclusión de todas las mujeres. Las jornadas "Cambio Educativo y Social III: Mujeres y Transformaciones sociales" ${ }^{\prime 4}$, celebradas en el Par-

2. FACEPA es la Federación de Asociaciones Culturales y Educativas de Personas Adultas.

3. El término de las Otras mujeres acuñado por Lídia Puigvert (2001) es sinónimo de mujeres sin estudios superiores, mujeres no académicas o mujeres populares. A lo largo de este artículo utilizaremos indistintamente estos términos para referirnos a este colectivo de mujeres. 
que Científico de Barcelona los días 23 y 24, han sido un espacio de debate entre mujeres no académicas y feministas con reconocimiento internacional como Judith Butler, Elisabeth Beck-Gernsheim y Lídia Puigvert.

\section{Aprendizaje dialógico, mujeres no académicas y educación}

Partimos del derecho que toda persona tiene a una educación de calidad. A partir de nuestra colaboración en centros de Educación de Personas Adultas y desde nuestro trabajo de investigación en CREA, Centro de Investigación Social y Educativa de la Universidad de Barcelona, hemos podido constatar que, a través de una educación de calidad, es posible superar la situación de exclusión que sufren determinados colectivos. Hemos tenido la oportunidad de comprobar que los centros de educación organizados de forma democrática y basados en los principios del aprendizaje dialógico (Flecha 1997) potencian la implicación de todas las personas, maximizan los aprendizajes y fomentan la transformación.

En la actual sociedad de la información, los aprendizajes realizados en contextos no-formales e informales están cobrando cada vez mayor importancia. Ésta es una de las principales razones por las que a nivel europeo se están priorizando investigaciones centradas en descubrir el valor de los conocimientos no académicos para manejarse en la sociedad de la información. Desde CREA, se está incidiendo en la importancia de transferir habilidades y competencias de contextos no-académicos a académicos y viceversa, así como desarrollar nuevas habilidades y competencias a través de las habilidades comunicativas ${ }^{5}$. Estas investigaciones señalan la importancia de acabar con las teorías de déficits (Flecha 1991) que han predominado en la educación de personas adultas, y que no hacen más que limitar el desarrollo de las capacidades que todas las personas poseemos.

Mientras nuestra reforma de la enseñanza se ha basado en concepciones obsoletas como el aprendizaje significativo y el constructivismo, la Educación de Personas Adultas a nivel mundial (Freire 1997b/1995) ha sido precursora del aprendizaje dialógico y la concepción comunicativa que hoy se está imponiendo en las comunidades científicas de educación y ciencias sociales.

De este modo hemos llegado a concluir que la participación de mujeres sin título universitario en Educación de Personas Adultas basada en el aprendizaje dialógico genera su protagonismo en la transformación social de las relaciones de género. Observamos como mujeres que han empezado a leer y escribir en centros de Educación de Personas Adultas organizados de forma democrática están ahora debatiendo sobre literatura clásica en espacios académicos junto a mujeres universitarias, liderando las reivindicaciones para la mejora de sus comunidades, y demandando relaciones más igualitarias en sus familias.

4. Las jornadas "Mujeres y transformaciones sociales" fueron organizadas por CREA recogiendo las demandas de las "Otras Mujeres" de FACEPA (Federación de Asociaciones Culturales y Educativas de Personas Adultas).

5. CREA. 1995-1998. Habilidades comunicativas y desarrollo social. DGYCIT, Dirección General de Investigación Científica y Técnica. Madrid. 
La alternativa que estamos desarrollando desde los centros de Educación de Personas Adultas dialógicos, a través del trabajo conjunto entre mujeres académicas y no académicas es el feminismo dialógico. Esta nueva perspectiva crítica aboga por la radicalización de la democracia en una apuesta por la capacidad de acción y reflexión de todas las personas. Sólo a partir del diálogo igualitario es posible incluir las reivindicaciones de todas las mujeres en el feminismo del siglo XXI, sin distinciones por cuestiones de clase social, nivel de estudios, edad o identidad cultural. El feminismo dialógico desarrolla los siete principios del aprendizaje dialógico: diálogo igualitario, inteligencia cultural, transformación, dimensión instrumental del aprendizaje, creación de sentido, solidaridad e igualdad de diferencias.

\section{a. Diálogo igualitario}

El diálogo es igualitario cuando considera las diferentes aportaciones en función de la validez de sus argumentos, en lugar de valorarlas por las posiciones de poder de quienes las realizan (Flecha 1997). Por este motivo, las mujeres que participan en centros de Educación de Personas Adultas demandan que los acuerdos se establezcan a través de un diálogo igualitario en el que todas puedan participar a través de sus ideas $y$ argumentos.

En la mayoría de centros de Educación de Personas Adultas, las mujeres populares son sometidas a una relación basada en la acción teleológica de un equipo de profesionales. Estas instancias escogen los mejores medios para conseguir sus fines como tener el horario más conveniente para el funcionariado. En esas condiciones, las aulas y los centros son concebidos como espacios para callar. El alumnado se convierte en consumidor de las ofertas formativas decididas desde arriba sin su participación.

En los centros de Educación de Personas Adultas de orientación dialógica (Cuenca 1998) las relaciones se basan en la acción comunicativa, es decir, en una interacción que reconoce a todos los sujetos su capacidad de lenguaje y acción y entablan una relación interpersonal. Todas las personas, incluyendo alumnado y comunidad, participan en el diseño de su aprendizaje. En esas condiciones, las aulas y los centros son concebidos como espacios para conversar. Las personas participantes son protagonistas de las ofertas formativas en cuya elaboración y decisión tienen una voz al mismo nivel que los profesionales y las profesionales.

Las mujeres debemos luchar para que vayan desapareciendo aquellos centros o asociaciones donde se silencia la voz de las mujeres no académicas e incluso se llega a impedir la organización autónoma de asociaciones de participantes o mujeres participantes.

Estas situaciones son las que las Otras mujeres han comenzado a crear y están llevando hacia el debate público, como en el pasado hicieron sólo las mujeres académicas. Son mujeres que luchan desde su vida cotidiana por construir un feminismo donde TODAS tengamos voz y acción y donde fomentemos la participación igualitaria en nuestro entorno social. 


\section{b. Inteligencia cultural}

Todas las personas tienen las mismas capacidades para participar en un diálogo igualitario. Aunque cada una puede demostrarlas en ambientes distintos, hay quienes lo hacen mejor en el mercado o en casa y sentir una inhibición total en un aula; hay quienes se desenvuelven bien en un ambiente académico y sin embargo, pueden verse inútiles en una reunión de vecinos o en la cocina.

El aprendizaje dialógico contempla como la pluralidad de dimensiones de la interacción humana y las habilidades comunicativas son componentes importantes de la inteligencia cultural. Se resuelven con ellas muchas operaciones que no lograría solucionar un actor por sí solo con sus inteligencias académica y práctica. Todas las personas tienen inteligencia cultural, la desigualdad se genera en sus diferentes desarrollos en entornos diversos. Unas hacemos bien exámenes de psicología evolutiva, otras saben interpretar el lenguaje de un bebé y responder a sus necesidades básicas. El tránsito de un ámbito a otro es posible siempre que quienes interactúan con las mujeres tengan el convencimiento de que pueden lograrlo y exista la oportunidad de demostrarlo.

CREA ha elaborado la concepción de inteligencia cultural. Entre sus subconjuntos están las inteligencias académica y práctica y también el resto de capacidades de lenguaje y acción de los seres humanos que hacen posible llegar a acuerdos en los diferentes ámbitos sociales. El concepto de inteligencia cultural ofrece un marco adecuado para superar todas las teorías de los déficits, incluidas las que se refieren de manera específica a la población adulta.

Las mujeres no académicas que participan en centros de orientación dialógica de Educación de Personas Adultas exigen, cada vez más, que sus habilidades y competencias sean valoradas por el mundo académico. Es mucho lo que tienen que aportar tanto a las mujeres con título universitario como al resto de personas y grupos.

\section{c. Transformación}

Está demostrado que el aprendizaje dialógico transforma las relaciones entre las personas y su entorno. Sin embargo, diferentes grupos de poder y teorías han puesto especial interés en tratar de convencer de la inutilidad de su esfuerzo a profesionales y movimientos que trabajan por lograr estas transformaciones. Evidentemente, estas transformaciones no tienen ningún valor para los autores reproduccionistas. Según ellos, como no cambia todo, no cambia nada y, en consecuencia, no es posible ninguna transformación real y niegan la posibilidad de mejora de personas como es el caso de las mujeres que asisten a centros de Educación de Personas Adultas.

Ningún estudio riguroso que analice el ámbito educativo puede ya prescindir de tener en cuenta tanto lo que reproduce como lo que transforma. La participación de mujeres populares en experiencias de aprendizaje dialógico va acompañada de importantes logros personales y sociales que desmienten día a día el modelo de la reproducción.

Frente a las profesoras y los profesores que quieren orientar sus cambios, estas mujeres quieren ser protagonistas de sus propios cambios y lo hacen desde sus colectivos y asociaciones. Llegando a acuerdos y contrastando sus diferencias, van conclu- 
yendo que nadie orienta a nadie, que su comunicación intersubjetiva orienta a todas (Puigvert 2001).

La comunicación horizontal entre mujeres académicas y populares genera un feminismo mucho más radical y profundo que el que es monopolizado por personas con título universitario que se creen portadoras de las ideas hacia donde tienen que caminar las transformaciones de todas las mujeres. Mientras tanto, muchas feministas o exfeministas tituladas, tras la crisis de sus opciones transformadoras tradicionales, se centran en la defensa de sus intereses corporativos e individuales.

Las transformaciones personales y sociales aumentan espectacularmente al participar en espacios de aprendizaje dialógico. Muchas mujeres empiezan a demandar que se les valore en las diferentes esferas en las que participan. La autoestima se incrementa cuando desde los centros de Educación de Personas Adultas se potencian sus capacidades. Las transformaciones de las que las Otras mujeres son sujetos rompen con la idea de que el sistema educativo reproduce las desigualdades sociales.

Rosa, una mujer popular que empezó a leer y escribir en un centro de Educación de Personas Adultas de orientación dialógica es en la actualidad licenciada en psicología, está estudiando el doctorado y trabajando de psicóloga. Esther, compañera de Rosa, después de obtener el título de graduado escolar se licenció en Historia y hoy dirige una cooperativa, a parte de dedicar el tiempo que le queda a su tesis doctoral. Mujeres como Rosa o como Esther, en su propósito de estudiar en la universidad siendo madres y amas de casa, nos muestran que la transformación no sólo es posible sino una realidad.

\section{d. Dimensión instrumental}

Desde la diversidad, muchas de las alternativas conservadoras y progresistas coinciden con frecuencia en su defensa a la oposición entre aprendizaje instrumental y aprendizaje dialógico. Posturas tradicionalistas condenan el excesivo diálogo en el aula y la democratización de la institución educativa como factores de la decadencia del aprendizaje técnico y científico. Al mismo tiempo, movimientos de renovación pedagógica proponen la formación humana en contraposición a la técnica, acercándose a propuestas educativas de tipo "laissez-faire". Diversas concepciones edistas se apuntan a esta propuesta en la Educación de Personas Adultas al considerar que, en la adultez, ya está deteriorada la capacidad para los aprendizajes instrumentales. Algunos autores llegaron incluso a enfrentar el aprendizaje dialógico con el instrumental.

El aprendizaje dialógico abarca todos los aspectos que unas personas o grupos acuerdan aprender. Incluye, por lo tanto, el aprendizaje instrumental de aquellos conocimientos y habilidades que se consideran necesarios saber. El aprendizaje dialógico no se opone al aprendizaje instrumental, sino a que los objetivos y procedimientos sean decididos al margen de las personas, justificándose con razones de tipo técnico que esconden los intereses exclusores de unas minorías académicas.

El aprendizaje instrumental se intensifica y profundiza cuando se sitúa en un adecuado marco dialógico. La capacidad de selección y procesamiento de información es el mejor instrumento cognitivo para desenvolverse en la sociedad actual y el diálogo fomenta el desarrollo de esa capacidad. La interacción con otras personas pone 
a nuestra disposición tanto informaciones diversas como su selección y procesamiento. Cuando el diálogo es igualitario se produce una intensa reflexión y un aprendizaje mediante la comprensión de los argumentos ajenos y la aportación de los propios.

Las mujeres que se embarcan en este tipo de aprendizajes buscan romper las barreras entre la esfera privada y pública, demandando en ambas la necesidad de relaciones igualitarias basadas en el diálogo. Muchas de las mujeres no académicas exigen la necesidad de una coherencia entre ambas esferas, coherencia que no han mostrado muchas mujeres académicas defensoras de la liberalización de la mujer y que, paradójicamente, se someten a relaciones de poder dentro de la esfera privada.

\section{e. Creación de sentido}

Todas las personas podemos soñar y dar sentido a nuestra vida. La aportación de cada una de ellas es diferente a la del resto $y$, por tanto, irrecuperable si no se tiene en cuenta. Cada persona excluida es una pérdida irremplazable para todas las demás. La creación de nuevos significados, a partir del diálogo igualitario, en las vidas de las mujeres es lo que potencia las transformaciones personales y sociales. La creación de sentido desde los centros de Educación de Personas Adultas se fomenta cuando los aprendizajes realizados dentro del aula guardan una conexión con el mundo de la vida de las personas participantes. A través del diálogo igualitario, las mujeres se enriquecen de las contribuciones de sus compañeras y buscan su propio sentido.

\section{f. Solidaridad}

Las prácticas educativas igualitarias sólo pueden fundamentarse en concepciones solidarias. La solidaridad entre mujeres se convierte en factor clave en la lucha por la transformación de las relaciones de género desiguales. Es posible observar los fuertes lazos de solidaridad entre mujeres en los centros de Educación de Personas Adultas. Gracias a esta solidaridad y ayuda muchas mujeres recobran la confianza en sí mismas, tanto en su capacidad para aprender como en sus posibilidades para transformar sus relaciones en el hogar y en otras esferas de la vida pública.

Las mujeres sin título universitario demuestran, desde la Educación de Personas Adultas, la importancia de la solidaridad para la construcción de un nuevo feminismo que incluya las voces de todas las mujeres. Para ellas, solidaridad significa la apuesta por las capacidades de todas las compañeras para la transformación personal y social. Es por ello, que las Otras mujeres están aportando al feminismo la necesidad de fomentar la autonomía a través de la solidaridad entre mujeres, evitando la dependencia de personas "expertas".

\section{g. Igualdad de diferencias}

Las reformas de la diversidad han generado desigualdades educativas. La mayoría de las propuestas educativas no han sabido superar el binomio igualdad-diferencia. Para superarlo, se requieren dos reorientaciones de la enseñanza: cambiar el objetivo de la diversidad por el de igualdad de diferencias y pasar de una concepción caduca de aprendizaje significativo al aprendizaje dialógico.

Sólo desde la igualdad de diferencias se defiende y se busca la igualdad de todas las personas y, por lo tanto, la igualdad de todas las mujeres. Se trata, en definitiva, de 
sentar las bases para crear unas condiciones más libres e igualitarias para el diálogo y el consenso entre todas.

El aprendizaje dialógico se orienta hacia la igualdad de diferencias afirmando que la verdadera igualdad incluye el mismo derecho de toda persona a vivir de forma diferente. El aprendizaje dialógico engloba y supera el significativo, aportando elementos para la superación de las desigualdades educativas. Con frecuencia, la igualdad homogeneizadora está relacionada con el objetivismo, la diversidad sin igualdad con una concepción obsoleta del constructivismo y la igualdad de las diferencias con una perspectiva dialógica.

La lucha por la igualdad de oportunidades es el objetivo principal del feminismo dialógico. Mujeres con identidades, edades y niveles académicos muy diferentes comparten espacios de diálogo en los centros de Educación de Personas Adultas. Las mujeres no académicas reivindican la necesidad de basar el feminismo en la igualdad de diferencias, es decir, en el igual derecho a ser diferentes.

\section{Experiencias de feminismo dialógico}

\subsection{ESREA (European Society for Research on the Education of Adults)}

ESREA nació en 1992 con el objetivo de basar su trabajo principalmente en la creación de redes universitarias de investigación, que en un principio fueron europeas y actualmente son a nivel mundial.

Desde su creación pretende establecer cooperación activa entre investigadoras y investigadores de todo el mundo que trabajen campos específicos en Educación de Personas Adultas desde sus diferentes universidades. Es pues, una asociación formada por universidades y centros de investigación académica.

ESREA funciona a partir de redes de trabajo (networks) a las que se vinculan académicas y académicos de diversas áreas de interés. Cada red de trabajo se autoorganiza independientemente del resto de grupos y acuerda una persona/grupo coordinador que propone y prepara las actividades de la misma. ESREA se reúne para compartir su trabajo intelectual a través de un congreso anual y de un comité de gestión que organiza y coordina los acuerdos tomados en las asambleas.

Actualmente son 14 las redes de trabajo que fomentan el intercambio y la creación de conocimiento científico; "Mujeres populares y educación" es una de ellas. Desde ESREA se entiende que parte de las investigaciones académicas que realizamos deben ir orientadas hacia el mercado, pero también se entiende que las sociedades no son sólo competitivas en el mercado, sino que están formadas por personas, ideas y sentimientos.

Por este motivo, consideramos que algunas de las investigaciones académicas de ESREA deben centrarse en procesos de transformación social con el fin de superar la exclusión social y reducir la distancia entre la tarea investigadora por un lado y la gente y los movimientos sociales por el otro. De este modo se abren nuevos canales de participación activa. 
La red de "Mujeres populares y educación" está formada con y para mujeres que poseen niveles académicos iniciales y pretende también aumentar la participación de investigadoras e investigadores que no hablan la lengua inglesa, apoyando su dimensión multilingüe.

Esta red quiere potenciar la actividad académico-investigadora en el área de Educación de Personas Adultas y promover el conocimiento de nuevas fórmulas de participación social de mujeres no académicas, reconociendo -mediante la investigaciónsu diversidad cultural.

Está encaminada para desarrollar teorías radicales que permitan la creación de canales de participación social y la radicalización de la democracia a través de la extensión de la educación y la formación durante toda la vida a mujeres con niveles académicos iniciales o sin ellos. Nos centramos en dos objetivos:

1. Desarrollar actividades de investigación sobre cómo abrir vías de participación social a mujeres que participan en educación popular.

2. Investigar sobre cómo las mujeres que son silenciadas, incluso en la mayoría de grupos feministas, pueden hacer oír su voz y fomentar su ciudadanía activa.

\subsection{El grupo de mujeres de FACEPA (Federación de Asociaciones Culturales y Educa- tivas de Personas Adultas)}

FACEPA es una organización formada por asociaciones y colectivos de participantes de centros de Educación de Personas Adultas y de entidades culturales nacida en el marco de actuación de Catalunya, aunque actualmente se encuentra en proceso de extensión al resto de autonomías.

Algunos de sus objetivos principales son: a) potenciar el acceso a los recursos educativos y culturales de todas las personas adultas, fundamentalmente de los sectores más desfavorecidos (emigrantes, mujeres, personas en paro,...) con la finalidad de combatir las desigualdades culturales y educativas; b) elaborar, mediante la práctica de los grupos federados, una alternativa concreta y abierta a la educación y a la participación cultural de las personas adultas, que tenga en cuenta todo tipo de iniciativas públicas, tanto institucionales como populares, caracterizadas por la falta de lucro, la gestión democrática, el pluralismo ideológico, la apertura al entorno y la calidad; c) impulsar un modelo de Educación de Personas Adultas participativo y social, en el que las personas participantes (estudiantes, asociaciones culturales y sociales) de la zona donde se desarrollan los proyectos intervengan en la definición y la gestión de los diferentes proyectos educativos y d) fomentar la solidaridad entre los diferentes grupos y asociaciones federadas.

Entre las actividades que desarrollan están: a) Carta de la Declaración de los Derechos de los Participantes: las personas participantes quieren definir y concretar unos derechos que les permitan gozar de una educación adaptada a sus necesidades e intereses y definida a través del acuerdo entre todos los agentes implicados en Educacción de Personas Adultas; b) Intercambio de experiencias: proporciona un espacio de reflexión sobre temas que preocupan y que son de interés común para las personas participantes y c) grupo de mujeres: encuentro y diálogo entre mujeres con y sin estu- 
dios académicos que crean espacios de debate público sobre la situación de sumisión de la mujer y reflexionan y actúan sobre formas de romper con ésta.

\subsection{La Asociación Gitana de Mujeres Drom Kotar Mestipen}

Esta asociación es un ejemplo de la lucha por la igualdad de diferencias de todas las mujeres. En ella participan mujeres payas y gitanas de todas las edades, formación y ocupación. La asociación pretende generar relaciones entre las mujeres que permitan superar las discriminaciones que recaen en las mujeres gitanas por su condición de mujer y de pertenencia a una minoría. Entre sus objetivos destaca la dedicación a la educación a lo largo de toda la vida de todas las mujeres. Ésta se presenta como la clave en la superación de las desigualdades que se producen en una sociedad desigual. A través del diálogo igualitario se produce un cambio en la percepción de los horizontes de las mujeres gitanas, que se amplían.

A partir de experiencias como ésta, se confirma la necesidad del aprendizaje y del feminismo dialógico para la transformación de las mujeres sin título universitario y para conseguir la igualdad de diferencias entre todas las mujeres. La clave está en que todas las mujeres de distintas culturas, edades y niveles culturales unan sus esfuerzos y trabajen en plano de igualdad desde los centros de Educación de Personas Adultas de orientación dialógica para conseguir los mismos objetivos -su inclusión y reconocimiento- desde la propia especificidad.

\section{Bibliografía}

BECK, V. 1998a. La sociedad del riesgo. Barcelona: Paidós. (Trabajo original publicado en 1986).

BECK,V. 1998b. ¿Que es la globalización? Barcelona: Paidós. (Trabajo original publicado en 1997).

BECK-GERNSHEIM, E; Butler, J; Puigvert, L. (2001). Mujeres y transformaciones sociales. Barcelona: El Roure.

BECK, U. Y BECK-GERNSHEIM, E. 1998. El normal caos del amor. Barcelona: El Roure. (Trabajo original publicado en 1997).

BEJAR, H. 1990. El ámbito íntimo. Privacidad, individualismo y modernidad. Madrid: Alianza Universidad.

BENHABID, S. y CORNELLA, D. (editores). 1990. Teoría Feminista y Teoría Crítica. Valenciana: Alfons el Magnànim.

CASTELLS, M. 1997-1998. La era de la Información. Vol. 1-2-3. Madrid: Alianza. (Trabajo original publicado en 1996-1997).

CREA (en proceso). Habilidades comunicativas y desarrollo social.

CREA. 1995. Participación y no participación en EPA. Generalitat de Catalunya.

CUENCA, J.M. 1998. Centro de EPA "La Verneda-Sant Martí". Un pequeño milagro cotidiano. Cuadernos de Pedagogía, no 265, p.p. 33 -40. Barcelona: Praxis.

ELBOJ, C.; FLECHA, R. 2000. "La educación de personas adultas en la sociedad de la información". Educación XX1, Núm 3. pp. 141-162. Madrid: UNED. 
FLECHA, R.; GÓMEZ, J.; PUIGVERT, L. 2001. Teoría Sociológica Contemporánea. Barcelona: Paidós.

FLECHA, R. 1997. Compartiendo palabras. Barcelona: Paidós.

FLECHA, R. 1991. "Desarrollo curricular de la educación de las personas adultas en el marco de la reforma de la enseñanza". Materiales para la Educación de Adultos. Enero 1991. Núm 1. pp.9-19. Canarias: Radio Ecca.

FREIRE, P. 1997a. Pedagogía de la autonomía. Madrid: Siglo XXI. (Trabajo original publicado en 1996).

FREIRE, P.1997b. A la sombra de este árbol. Barcelona: El Roure. (Trabajo original publicado en 1996).

GIDDENS, A. 1995a. La Transformación de la intimidad. Cambridge: Polity Press. (Trabajo original publicado en 1992).

GIDDENS, A.1995b. Modernidad e Identidad del Yo. (Trabajo original publicado en 1991).

GIROUX, H. 1991. Postmodernism, Feminism, and Cultural Politics. Albany: State University of New York.

HABERMAS, J. 1987-1989. Teoría de la Acción Comunicativa. vols.I-II. Madrid: Taurus. (Trabajo original publicado en 1981).

PUIGVERT, L. 2001. Las otras mujeres. Barcelona: El Roure.

SÁNCHEZ, M. 1999. "La Verneda.St. Martí: School where people dare to dream". Harvard Educational Review, vol. 69.Núm. 3, pp. 320-335. Cambridge: Harvard University.

SOLER, M. 2001. Dialogic Reading. A new understanding of the reading event. Presentada en Harvard University. Tesis doctoral no publicada.

SUBIRATS, M. 1998. Con diferencia. Las mujeres frente al reto de la autonomía. Barcelona: Icaria.

SUBIRATS, M. 1997.“Notas para la búsqueda de una metodología de cambio desde los stereotipos de género". A Ensayos de Pedagogía Crítica. Madrid: Popular.

TOURAINE, A. 1997. ¿Podremos vivir juntos?. Iguales y diferentes. Madrid: PPC. 